\title{
TRIMOD MODULAR FORMATION ASSEMBLY USING "MARS" MODULAR ROBOTIC DEVICES
}

\author{
NIKITA PAVLIUK, PETR SMIRNOV AND ANTON SAVELIEV \\ St. Petersburg Institute for Informatics and Automation \\ of the Russian Academy of Sciences, 39, 14th Line, 199178, St. Petersburg, Russia \\ E-mail: antei.hasgard@iias.spb.su \\ http://robotics.nw.ru/
}

\begin{abstract}
Problems of configuration of homogeneous robot groups into three-dimensional spatial shapes are relevant, extensively researched and implemented in practice. This paper presents conceptual formations and results of modeling of mobile autonomous reconfigurable system, modular robotic unit and TriMod formation, as the basic spatial structure of the modular robotic system. Within the present research existing modular robotic systems were analyzed, isolated nodes, components and the issues of their pairwise connections. The analysis allowed to define a list of requirements to modular robotic devices and outline unresolved problems in this domain. There is currently no suitable model of autonomous robotic device and source formations, that would enable implementation of the established solution for configuration of a mobile robot group into the TriMod formation. The assessment of the solution in a simulation environment showed, that the proposed model outperforms similar solutions in many ways or enables alternative approaches to solve already known problems, as it has the following advantages: full-blown autonomous mobility system, self-centering mechanical device for connection with analogous modular devices, as well load-bearing capacity up to $3 \mathrm{~kg}$.
\end{abstract}

\section{Introduction}

Combined action of robotic devices within a single spatial structure allows to build mobile robotic device formations of different shapes, sizes, mobility modes, with various sensory and functional capabilities to solve a specialized problem in question. One of the challenges, related to configuration of a robotic device group consists in the overall complexity of robot device interconnection. Besides that, additional external conditions may have to be established to implement the whole process. In this paper a solution is proposed for autonomous assembly of robotic formation using homogeneous devices. A model of a modular robotic device is presented, as well a TriMod assembly, where three devices are connected into a composite formation, that can move as a whole unit. The research problem and the relevant scientific context are more thoroughly presented is the following related work.

One of the most difficult tasks in the design of modular robotic systems is to create a reliable and flexible connection mechanism. The various types of modular robotic tools offered today are characterized by a number of problems: different standards and methods of interaction between sensors and actuators, low reliability of structures and connection elements, and high power-demanding. The most important developments in this domain are the following ones:

In [1] the M-Block model is presented. M-Block is a modular, self-reconfiguring cubic robot with a magnet bonding, that use inertial forces to move independently in a range of environments. The modules achieve these movements by quickly transferring angular momentum accumulated in a self-contained flywheel to the body of the robot.

Mori robot [2] has a low-profile triangular structure with a manual engagement mechanism and a folding actuation mechanism at each edge. It is mobile on flat surfaces, can be attached to other modules of its kind and fold into any 3D configuration desirable. Each Mori robot controls the movement of three adjacent modules and can be folded into a pyramidal formation or opened 
arbitrarily. Internal components of the device require an external power source. The ChainRORM [3] robot can move by "worm movement", after the modules are manually assembled into a serpentine structure. The paper [4] presents a modular robot M TRAN III, a 3D-printed device. Each section consists of two articulated mobile phalanges. MTRAN III can form chain-like and tetrapod formations.

In [5] Tetrobot robot has rods that extend or shorten to achieve the desired modular structure shape. Tetrobot is a type of parallel robots with redundant connections and features a concentric multilink spherical joint which allows an arbitrary number of links io share a common center of rotation. The mechanism proposed in [6] ensures an efficient and high strength connection due to non-back drivable actuation and specially designed clamping profiles that enables modules to tolerate large loads, and the entire system to discard faulty modules, thereby increasing their versatility and reliability.

The paper [7] presents the design concept of a modular robot UBot. A novel modular selfreconfigurable robot called UBot is presented. The UBot module is compact, strength, flexible and capable of performing efficient locomotion, self-reconfiguration and manipulation tasks.

The self-reconfigurable robotic system Roombots [8] is developed for further application in the design of modular and reconfigurable furniture. Robots can move and interact with each other as well with things indoors in a prepared and well-structured environment, using mechanical connection devices. Some motions are also ensured via rotational movement of actuators. In a non-structured environment, robots can move, using only rotational motions. Mona [9] is an open-source and open-hardware mobile robot that has been developed at the University of Manchester. The low cost of the platform means that it is feasible for a large number of these robots to be used in swarm robotic scenarios.

A self-assembly modular robot SambotII [10] is developed based on SambotI, which is a previously built hybrid type self-modular robot that is capable of autonomous movement and self-assembly.

A review of related works shows the main disadvantages of the presented solutions: limited ability to interact with environment and low autonomy of modular robotic devices not in the formation. To solve the problem of limited autonomous movement and connection in the formation, model of homogeneous robotic system was developed. This model includes three types of main components: chassis for fully autonomous movement, connector and functional two-axis actuator as the central mechanism of the device, which rotates and bends relative to the geometric center of the device. Such a model provides the movement of modules, both together and separately. Further let us consider the TriMod formation in greater details.

\section{Model and Description of the TriMod Formation}

Compared to the considered analogues, the MARS model [11-13] has broad motion capabilities and stability as an autonomous device (see Figure 1, a). It can self-assemble into a formation, independently from the device positions in field before the formation configuration begins, as well to move as such formation. Each device is equipped with a battery and requires no external power supply. The connection mechanism of a modular robotic device (MRD) ensures autocentering of the devices in the connection process. TriMod formation (see Figure 1, b) consists of three devices, two of which are connected in perpendicular fashion into a H-form structure (with a central module). This allows to build a modular robotic platform, which may be used as a load bearing device, as well as a base unit for a configuration of a more complex formation with a greater number of devices and a broader functionality. 


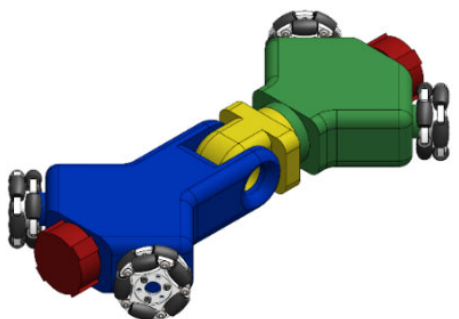

a

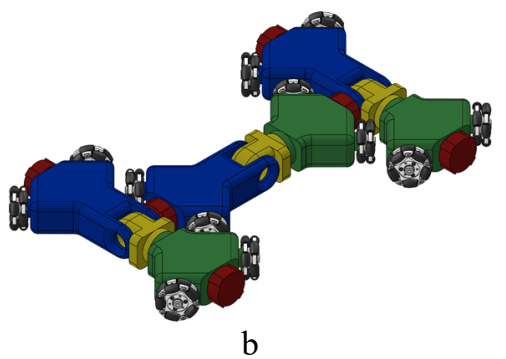

b

Figure 1. Models of a modular robotic device MARS (a) and TriMod formation (b).

MARS model is developed to solve mobility-related and autonomy-related problems, arising in practical applications of robotic devices. It also shows complex structures, composed of such devices. A basic formation TriMod is developed, assembled of a minimum number of modular robots. The formation is developed in such manner, that addition of extra devices to it extends the functionality of the obtained structure. To illustrate this, further we present simulation modeling, as well real-world modeling.

\section{Simulation Modeling}

To test the obtained model and formation, experiments in Gazebo simulation environment were performed. In the course of the TriMod structure formation, three robot models were used within a field. The size of the field was taken to be $3 \times 3 \mathrm{~m}$. In Figure 2 the TriMod formation process in the plane is presented. The modules sequentially move to the connection points, without causing collisions, and assemble into the specified configuration. During the modeling process 10 experiments were performed in TriMod formation assembly.
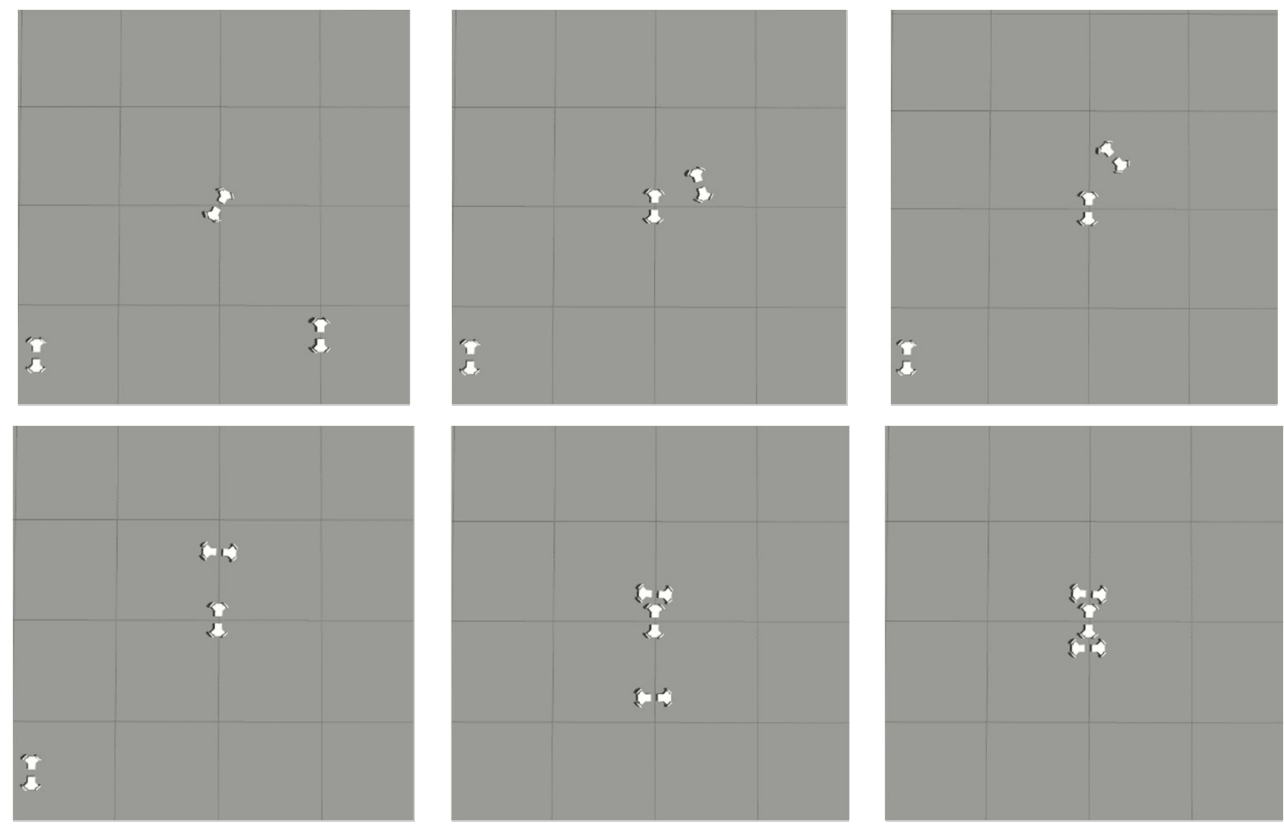

Figure 2. TriMod formation establishment.

Simulation modeling was performed as the following sequence of operations: 
1. Positioning of devices in the field in a random fashion.

2. Device control server initialization.

3. Device initialization.

4. Benchmarking of the baseline begin time.

5. Device movement with further composition into the formation.

6. Reconfiguration into the initial state of the formation.

7. Benchmarking of the baseline end time.

8. Logging of the obtained data.

The simulation modeling was performed to confirm the operability of the MARS system and estimation of average action time of the system. Average motion time, needed for all MRDs to reach their connection points was $144.7 \mathrm{sec}$. Total performance time of the system, including the motion of the established configuration, was $167.4 \mathrm{sec}$. These results are good enough and comply with our expectations.

\section{MRD Prototype, Comparison to the Analogous Devices}

For the modular robotic device model, a wheelbase on omni wheels is developed, allowing the MRD to turn on its heels and move diagonally. A self-centering mechanical connection device (MCD) is developed. An axial unit concept is developed, ensuring bending and axial rotation of the device relative to its geometric center within the formation. The characteristics of the prototype device are given in Table 1.

Table 1. MRD MARS characteristics.

\begin{tabular}{|c|c|}
\hline Device name & MRD MARS v1.2 \\
\hline Master microcontroller & ESP32-WROOM \\
\hline Battery & $900 \mathrm{mAh}$ \\
\hline Performance duration & Up to 40 minutes \\
\hline Weight & $1.1 \mathrm{~kg}$ \\
\hline Load capacity & $3 \mathrm{~kg}$ \\
\hline Dimensions & $340 \times 140 \times 50 \mathrm{~mm}$ \\
\hline Wheelbase & $\begin{array}{l}\text { Two pairs of own wheels, positioned according the classical pattern, using } \\
\text { holonomic motion, spaced from each other to install the central MRD mechanism } \\
\text { and two connection ports }\end{array}$ \\
\hline Axial unit & $\begin{array}{l}\text { Two-axial actuator, which ensures rotation and bending of the device relative to } \\
\text { the geometric center of the MRD and relative to two connection ports }\end{array}$ \\
\hline Connection device & $\begin{array}{l}\text { A mechanical one - active connection mechanism, implemented as a three-step } \\
\text { telescopic gripper }\end{array}$ \\
\hline Sensors & Infrared sensors of coaxial positioning \\
\hline
\end{tabular}

Mechanical connection device is implemented as a sliding gripper, based on an iris diaphragm [12]. The device has two main operating modes: 1) gripper; 2) connecting plate. The device was developed as a three-step telescopic mechanism with the following states:

1. Closed state.

2. Open state.

3. Connection plate grip.

In closed state the external outlines of the actuator reflect the shape of the connection plate, intended for grip in the course of formation establishment. The transition from the open state to the state of connection plate takes place also in process of formation, by gripping and fixed positioning of two MRDs. When plugging the units with each other a dovetail connection is 
established with a trapezoid angle of $45^{\circ}$. The design of the connection device includes infrared sensors, utilized for coaxial alignment of MRDs relative to each other. At distance up to $40 \mathrm{~mm}$ the sensors ensure recognition capabilities along the horizontal axis with error margin up to 12 $\mathrm{mm}$. Deflection along the vertical axis is excluded due to design features and the conditions of formation establishment. The actuators of the connection device are changeable and selfcentering, they allow for up to $15 \mathrm{~mm}$ error of mutual coaxial alignment of the devices. The axial unit of MRD is assembled as a two-axial actuator, rotating along two axes within $180^{\circ}$ along each, whereas the position of $90^{\circ}$ is considered the initial state. The MRD with such functional capabilities can be used at any point of the formation. The MRD MARS prototype is shown in Figure 3.

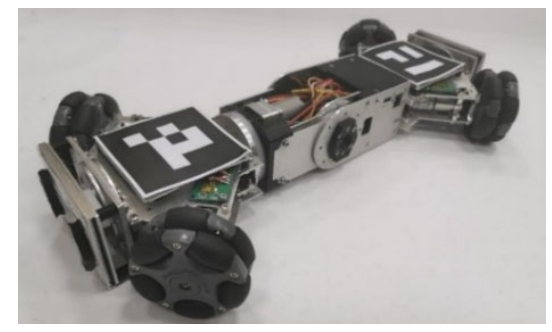

Figure 3. MRD MARS prototype.

Table 2 compares the characteristics of the assembled prototype with similar existing devices.

Table 2. Comparison of other existing modular robots and MARS.

\begin{tabular}{|c|c|c|c|c|}
\hline Model & Power supply & $\begin{array}{l}\text { Autonomous device } \\
\text { motion }\end{array}$ & $\begin{array}{l}\text { Device } \\
\text { connection } \\
\text { mode }\end{array}$ & Functionality of individual modules \\
\hline ChainFORM & External & No & Manual & LED indication \\
\hline M-Block & Battery & Yes & Magnetic & - \\
\hline Mori & External & Yes & Manual & - \\
\hline M-TRAN & Battery & Yes & Mechanical & Device bending and unbending \\
\hline Tetrobot & External & No & - & - \\
\hline UBot & Battery & Yes & Mechanical & Rotational joint \\
\hline Roombots & Battery & Yes & Mechanical & $\begin{array}{l}\text { Two spheres with mobile } \\
\text { hemispheres and connection devices }\end{array}$ \\
\hline Mona & Battery & Yes & - & - \\
\hline SambotII & Battery & Yes & Mechanical & $\begin{array}{l}\text { Autonomous connection with other } \\
\text { devices }\end{array}$ \\
\hline MARS & Battery & Yes & Mechanical & $\begin{array}{l}\text { Bending and unbending, axial } \\
\text { centered motion, autonomous } \\
\text { connection with other (identical) } \\
\text { devices, load bearing up to } 3 \mathrm{~kg} \text {. }\end{array}$ \\
\hline
\end{tabular}

A distinctive feature of the MRD MARS is the load-bearing capacity up to $3 \mathrm{~kg}$, what is three times heavier as the device itself. Autonomy and functionality of the device excludes the need of the of constant manual control of motion and connection mechanisms in the course of formation establishment and reconfiguration into the initial state. 


\section{Prototype Testing}

The presented prototype device is intended solely as proof of concept, to show the feasibility of the presented ideas in practice. It requires significant engineering improvement for any industrial application. So, let us consider the tests where research prototypes were employed to get an overall understanding of the problems to be solved. Prototype testing was performed in three steps, beginning from the operability check of the individual device at the first stage to the complete configuration of the TriMod formation. Tables 3 show key parameters of the test field, used in the experimental assembly of MRD TriMod.

Table 3. Parameters of test field $F$.

\begin{tabular}{cc}
\hline Field size, $F$ & $3 \times 3 \mathrm{~m}$ \\
\hline Field segment size, $S_{F}$ & $1,8 \times 1,8 \mathrm{~m}$ \\
MRD positioning in the field, $M_{O P}$ & Random \\
MRD outer dimensions, $G$ & $0,14 \times 0,34 \times 0,05 \mathrm{~m}$ \\
Number of the devices in the experiment, $M$ & 3 \\
\hline
\end{tabular}

The field of $3 \times 3$ meter (see Figure 4) suffices for random placement of $M_{O P}$ MRD and autonomous formation establishment. The formation establishment control is performed upon data, obtained from dour cameras, installed above the test field and tracking the position and orientation of each MRD in this field. The formation position control in the work area is performed with external cameras, allowing to detect augmented reality markers on MRD. Because robot position relative to each other is known, the position and orientation of the whole formation is also known, even if only one device is in the scope of camera. Test field contains augmented reality markers for calibration of control system cameras. The field is divided in four segments for complete coverage by cameras, tracking the MRD positions. The image, obtained from cameras, contains the whole field, ensuring constant visual control of MRD position and position adjustment if necessary. Resolution capacity of the cameras, used in the tests, was averaged according to the test field segment size.
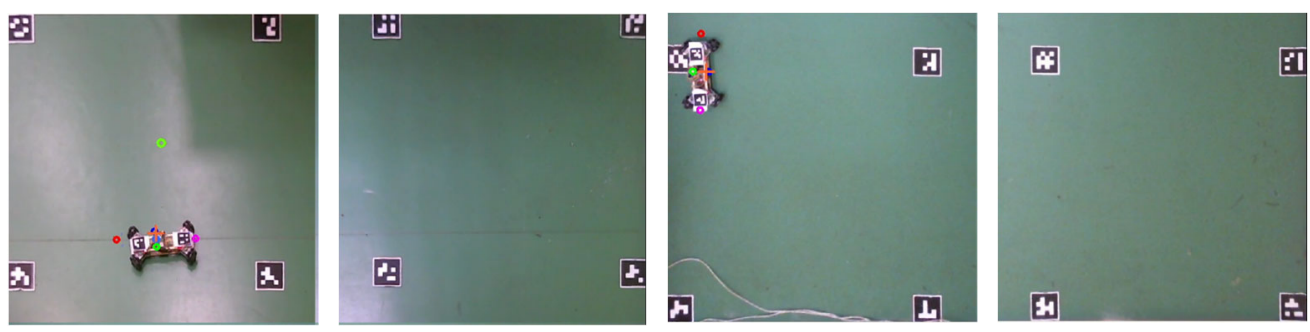

Figure 4 . Test field with the markers of augmented reality.

Also, segment overlapping in the viewport was respected to reduce the number of MRD tracking errors in the field, while the devices move from one segment to another. Cameras, installed above the test field, are calibrated after activation, and detect MRDs upon the installed augmented reality markers, which contain data on field marker positions and device identifiers. Compliance to this sequence of operations during MARS testing ensures comprehensiveness of the obtained data, as well timely revealing, and correction of functional system errors. At the first stage of the testing the function of one MRD was checked, as well time to reach the given 
position from the random initial points in the test field. The destination point was in the middle of the field, also corresponding to the central point of the TriMod formation.

In the second stage of the testing perpendicular connection of these MRDs was assessed, according to the TriMod formation sequence. Both devices move to the destination point from the random points in the field. To test the connection mechanism and infrared sensors, ensuring coaxial alignment and MRD positioning relative each other during connection process, the initial position of MRD in the field was chosen in such manner, that the MRD, moving to its destination point, would perform a $90^{\circ}$ turn.

In the third stage of the testing the composition of three randomly spaced MRDs into TriMod formation was assessed. To do this, the following destination points for the MRDs were set: the first point corresponded to the geometric center of the TriMod being assembled, the other two points corresponded to the positions of two more devices, that attach to the central one from the sides. The central device is designated the one, that is the closest to the geometric center of the formation, whereas the two other devices adjust their positions according to the optimal path tracing algorithm.

Fifty experiments with three MRD prototypes were performed to assess the quality of TriMod formation establishment (see Figure 5) on the test field.
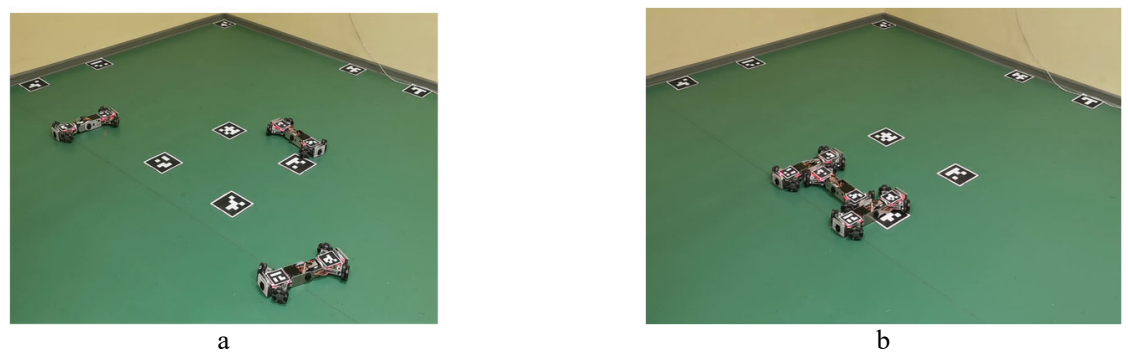

Figure 5. Testing of the TriMod formation: a) initial position of the formation; b) final position of the formation.

The first testing stage included 10 runs of an individual MRD from different points of the test field, average system performance duration was $33 \mathrm{sec}$, average time to reach the destination point for the MRD was $18 \mathrm{sec}$. Average distance, covered by the MRD, was $1.2 \mathrm{~m}$. System performance measurements included the timeframes, needed for initialization and calibration of all devices.

The second testing stage, based on 7 experiments, showed average system operation time of $96 \mathrm{sec}$, average MRD motion time to the connection position was $56 \mathrm{sec}$. The process of coaxial alignment calibration, as well connection of the ports of central and perpendicularly positioned devices required less than $10 \mathrm{sec}$.

The third testing stage, based on 33 experiments with prototypes, aimed to assemble the TriMod formation, showed, that the average time, required for all the MRDs to reach the connection positions, was $134 \mathrm{sec}$. Total system performance time, including the motion of the assembled TriMod configuration, was $169 \mathrm{sec}$.

Additionally, 19 experiments were performed to test MRD motion within a whole formation (see Figure 5, b). During MARS prototype tests the MRD positioning accuracy upon cameras was increased, specifically: camera calibration was performed in real-time mode during test process, what allowed to reduce the robot positioning error from $15-25 \mathrm{~mm}$ to $10-15 \mathrm{~mm}$. Also, the accuracy of mutual MRD positioning at connection step was increased via choosing of minor distance to be covered per iteration step (from 20 to $10 \mathrm{~mm}$ ). Hence, in the first testing 
stage we confirmed, that the device can successfully reach its destination point and rebuild itself into the required formation. This task was successfully completed.

Further we confirmed good interoperability of MRDs with each other, particularly, their fitness to assemble into the predefined formations. Concerning overall performance time of the system, that was investigated on the third stage of the experiments, the whole experimental workflow, described above, initially required $220 \mathrm{sec}$ in average to be completed. Though, after algorithm and code optimization, this average time was reduced to $169 \mathrm{sec}$, what is a major improvement.

\section{Conclusion}

The proposed solution provides for configuration of homogeneous robotic devices into various formations, thereby extending the range of tasks, that could be performed by them, increasing the load-bearing capacity and mobility of the robotic vehicles via their reconfiguration based on the developed basic formation. The proposed model of a modular robotic device allows to offload the computing control unit into a dedicated pluggable device with a standard set of connection, power supply and switching interfaces, what further allows to adjust the computing power of the system if necessary. The results of the research, as well the designed prototypes, can be applied in the domain of cyber-physical systems [14-16].

\section{Acknowledgments}

This research is supported by the RFBR Project No. 20-08-01109.

\section{References}

1. J.W. Romanishin, K. Gilpin and D. Rus, IEEE Intern. Conf. on Intel. Robots and Systems. Art. no. 6696971, 42884295 (2013).

2. C. H. Belke and J. Paik, IEEE/ASME Trans. on Mech. 22(5), 2153-2164 (2017).

3. K. Nakagaki, et al., In Proc. of the 29th Annual Symp. on User Inter. Software and Tech. 87-96 (2016).

4. S. Murata, et al., IEEE/ASME Trans. on Mech. 7(4), 431-441 (2002).

5. G. J. Hamlin and A.C. Sanderson, Proc. of 1995 IEEE Inter. Conf. on Robotics and Automation. 1, 154-159 (1995).

6. W. Saab and P. Ben-Tzvi, ASME 2015 Inter. Design Engin. Tech. Conf. and Comp. and Inform. in Engin. Conf. American Society of Mech. Engin. V05BT08A007-V05BT08A007 (2015).

7. S. Tang, et al., 2009 ASME/IFToMM Intern. Conf. on Reconf. Mech. and Rob. 529-535 (2009).

8. S. Hauser, M. Mutlu, P. A. Léziart, H. Khodr, A. Bernardino, A. J. Ijspeert, Robotics and Autonomous System. 127 (2020).

9. F. Arvin, et al., UK-RAS Conf. on 'Robotics and Autonomous Systems. 49-52 (2017).

10. W. Tan, H. Wei and B. Yang, App. Sci. 8(10), 1719 (2018).

11. N. Pavliuk, D. Pykhov, A. Saveliev and E. Cherskikh, Smart Innov., Syst. and Tech. 154, 383-395 (2020).

12. N. Pavliuk, P. Smirnov, A. Kondratkov and A. Ronzhin, Intern. Conf. on Inter. Collab. Rob. 260-269 (2019).

13. N. Pavliuk, K. Krestovnikov and D. Pykhov, Problemele Energeticii Regionale. 125-135 (2018).

14. I. Vatamaniuk and R. Iakovlev, Proc. of S-W State Univ. 23(6), 225-240 (2019).

15. R. Iakovlev and I. Vatamaniuk, Mod., Optimiz. and Inform Tech. 7(4) (2019).

16. R. Iakovlev, I. Vatamaniuk and D. Malov, 12th Intern. Conf. on the Develop. in eSystems Engin. (2019). 